\title{
The Intrinsic Structure and Properties of Laplace-Typed Integral Transforms
}

\author{
Hwajoon Kim \\ Faculty of General Education, Kyungdong University, Yangju, Gyeonggi 11458, Republic of Korea \\ Correspondence should be addressed to Hwajoon Kim; cellmath@gmail.com
}

Received 14 February 2017; Revised 12 April 2017; Accepted 10 May 2017; Published 8 June 2017

Academic Editor: Salvatore Strano

Copyright (c) 2017 Hwajoon Kim. This is an open access article distributed under the Creative Commons Attribution License, which permits unrestricted use, distribution, and reproduction in any medium, provided the original work is properly cited.

We would like to establish the intrinsic structure and properties of Laplace-typed integral transforms. The methodology of this article is done by a consideration with respect to the common structure of kernels of Laplace-typed integral transform, and $G$-transform, the generalized Laplace-typed integral transform, is proposed with the feature of inclusiveness. The proposed Gtransform can provide an adequate transform in a number of engineering problems.

\section{Introduction}

The key motivation for pursuing theories for integral transforms is that it gives a simple tool which is represented by an algebraic problem in the process of solving differential equations [1]. In the most theories for integral transforms, the kernel is doing the important role which transforms one space to the other space in order to solve the solution. The main reason to transform is because it is not easy to solve the equation in the given space, or it is easy to find a characteristic for the special purpose. For example, in computed tomography (CT) or magnetic resonance imaging (MRI), we obtain the projection data by integral transform and produce the image with the inverse transform. This is the strong point of integral transform.

To begin with, let us see the intrinsic structure of Laplacetyped integral transforms. Of course, the structure is dependent on the kernel, and the form of the kernel in Laplacetyped integral transforms is as follows. Laplace transform is defined by

$$
\mathfrak{E}(f)=\int_{0}^{\infty} e^{-s t} f(t) d t
$$

Sumudu one is

$$
S(u)=\frac{1}{u} \int_{0}^{\infty} e^{-t / u} f(t) d t,
$$

and Elzaki one is

$$
E(u)=u \int_{0}^{\infty} e^{-t / u} f(t) d t .
$$

Since the Laplace transform $\mathfrak{E}(f)$ can be rewritten as

$$
\int_{0}^{\infty} e^{-t / u} f(t) d t
$$

for $s=1 / u$, we can naturally consider that the form of Laplace-typed integral transform $G$ is

$$
F(u)=G(f)=u^{\alpha} \int_{0}^{\infty} e^{-t / u} f(t) d t
$$

for an suitable integer $\alpha$ : for example, if

$$
G(1)=u^{\alpha} \int_{0}^{\infty} e^{-t / u} d t=u^{\alpha}(-u)\left[e^{-t / u}\right]_{0}^{\infty}=u^{\alpha+1}
$$

for $u>0$.

Normally, integral transforms have a base of exponential function and it gets along with how to integrate from 0 to $\infty$ in order to utilize the property to $e^{-s t}$ or $e^{-t / u}$ converges to 0 when $t$ approaches $\infty$. Sumudu/Elzaki transform is a kind of modified Laplace one introduced by Watugala [2] in 1993/Elzaki et al. [3] in 2012 to solve initial value problems in engineering problems [4]. Belgacem et al. [5, 6] 
are mentioning that Sumudu transform $(\alpha=-1)$ has scale and unit-preserving properties, and it may be used to solve problems without resorting to a new frequency domain. Elzaki et al. $[3,7,8]$ insists that Elzaki transform $(\alpha=1)$ should be easily applied to the initial value problems with less computational work and solve the various examples which are not solved by the Laplace or the Sumudu transform. As an application, Agwa et al. [9] deal with Sumudu transform on time scales and its applications, Eltayeb and Kilicman [10] have checked some applications of Sumudu one, and Eltayeb et al. are highlighting the importance of fractional operators of integral transform and their applications in [11]. The shifted data problems, shifting theorems, and the forms of solutions of ODEs with variable coefficients can be found in $[4,12,13]$.

On the other hand, Kreyszig [1] says that Laplace transform $(\alpha=0)$ has a strong point in the transforms of derivatives; that is, the differentiation of a function $f(t)$ corresponds to multiplication of its transform $\mathfrak{E}(f)$ by $s$. In the other view, if we want the inverse case, the transform giving a simple tool for transforms of integrals, then we can choose a suitable form of integral transform such as

$$
\frac{1}{u^{2}} \int_{0}^{\infty} e^{-t / u} f(t) d t
$$

This means that the integer $\alpha$ is applicable to -2 . As we checked above, the comprehensive transform in Laplacetyped ones is needed, and thus we would like to propose $G$ transform, a generalized Laplace-typed integral transform, which is more comprehensive and intrinsic than the existing transforms.

This intrinsic structure in Laplace-typed integral transforms has a meaning which can be directly applied to any situation by choosing an appropriate integer $\alpha$. The main objective of this paper is to construct the generalized form of Laplace-typed integral transforms and establish the properties of it, and, to the author's knowledge, the proposed $G$-transform is the first attempt to generalize Laplace-typed integral transforms. Finally, we would like to mention that Laplace transform gave many considerations to this article.

\section{The Properties of Laplace-Typed Integral Transforms}

2.1. The Definition and the Table of Generalized Integral Transform G, Shifting Theorems. As mentioned before, let us rewrite the definition of Laplace-typed integral transforms, and we would like to call it $G$-transform.

Definition 1. If $f(t)$ is an integrable function defined for all $t \geq 0$, its generalized integral transform $G$ is the integral of $f(t)$ times $u^{\alpha} \cdot e^{-t / u}$ from $t=0$ to $\infty$. It is a function of $u$, say $F(u)$, and is denoted by $G(f)$; thus

$$
F(u)=G(f)=u^{\alpha} \int_{0}^{\infty} e^{-t / u} f(t) d t
$$

Let us first check the shifting theorems.
Theorem 2. (1) (u-shifting) If $f(t)$ has the transform $F(u)$, then $e^{a t} f(t)$ has the transform

$$
F\left(\frac{u}{1-a u}\right)
$$

That is,

$$
G\left[e^{a t} f(t)\right]=F\left(\frac{u}{1-a u}\right)
$$

(2) (t-shifting) If $f(t)$ has the transform $F(u)$, then the shifted function $f(t-a) h(t-a)$ has the transform $e^{-a / u} F(u)$. In formula,

$$
G[f(t-a) h(t-a)]=e^{-a / u} F(u)
$$

for $h(t-a)$ is Heaviside function (we write h since we need $u$ to denote $u$-space). Additionally, $G[h(t-a)]=u^{\alpha+1} e^{-a / u}$ holds.

Proof. (1) From

$$
\begin{aligned}
G\left[e^{a t} f(t)\right] & =u^{\alpha} \int_{0}^{\infty} e^{-t / u} e^{a t} f(t) d t, \\
e^{-t / u} e^{a t} & =e^{(a-1 / u) t}=e^{-t /(u /(1-a u))},
\end{aligned}
$$

we obtain the result.

(2)

$$
\begin{aligned}
e^{-a / u} F(u) & =e^{-a / u} \cdot u^{\alpha} \int_{0}^{\infty} e^{-\tau / u} f(\tau) d \tau \\
& =u^{\alpha} \int_{0}^{\infty} e^{-(a+\tau) / u} f(\tau) d \tau .
\end{aligned}
$$

Substituting $\tau+a=t$, we obtain

$$
\begin{aligned}
e^{-a / u} F(u) & =u^{\alpha} \int_{a}^{\infty} e^{-t / u} f(t-a) d t \\
& =u^{\alpha} \int_{0}^{\infty} e^{-t / u} f(t-a) h(t-a) d t \\
& =G[f(t-a) h(t-a)]
\end{aligned}
$$

for $h$ is Heaviside function.

By the similar way, we have $G[h(t-a)]=u^{\alpha+1} e^{-a / u}$ for $h$ is Heaviside function.

Using $G(f)=u^{\alpha} \cdot F(1 / u)$ for Laplace transform $\mathfrak{E}(f)=$ $F(s)$, we can obtain the table of generalized integral transform $G$ as shown in Table 1. In the table, we regard Laplacetyped integral transform $G$ as a transform. However, we can choose an appropriate constant according to each situation. For example, the choice of $\alpha=0$ has a merit in the transforms of derivatives, and $\alpha=-2$ has a strong point in the transforms of integrals. 
TABLE 1: Table of Laplace-typed integral transform $G$.

\begin{tabular}{lcc}
\hline & $f(t)$ & $G(f)$ \\
\hline 1 & 1 & $u^{\alpha+1}$ \\
3 & $t$ & $u^{\alpha+2}$ \\
4 & $t^{n}$ & $n ! \cdot u^{n+\alpha+1}$ \\
5 & $e^{a t}$ & $\frac{u^{\alpha+1}}{1-a u}$ \\
6 & $\sin a t$ & $\frac{a u^{\alpha+2}}{1+u^{2} a^{2}}$ \\
7 & $\cos a t$ & $\frac{u^{\alpha+1}}{1+u^{2} a^{2}}$ \\
8 & $\sinh a t$ & $\frac{a u^{\alpha+2}}{1-u^{2} a^{2}}$ \\
9 & $\cosh a t$ & $\frac{u^{\alpha+1}}{1-u^{2} a^{2}}$ \\
10 & $e^{a t} \cos b t$ & $\frac{u^{\alpha}(1 / u-a)}{(1 / u-a)^{2}+b^{2}}$ \\
& $e^{a t} \sin b t$ & $\frac{b u^{\alpha}}{(1 / u-a)^{2}+b^{2}}$ \\
\hline
\end{tabular}

If $f(t)$ is defined and is piecewise continuous on $t \geq 0$ and satisfies $|f(t)| \leq M e^{k t}$ for all $t \geq 0$, then $G(f)$ exists for all $u<1 / k$. Since

$$
\begin{aligned}
|G(f)| & =\left|u^{\alpha} \int_{0}^{\infty} e^{-t / u} f(t) d t\right| \\
& \leq u^{\alpha} \int_{0}^{\infty} e^{-t / u}|f(t)| d t \leq u^{\alpha} \int_{0}^{\infty} e^{-t / u} M e^{k t} d t \\
& =\frac{M u^{\alpha+1}}{k u-1}
\end{aligned}
$$

the statement is valid.

\subsection{Transforms of Derivatives and Integrals}

Theorem 3. Let a function $f$ be $n$-th differentiable. Then the transforms of the first, second, and $n$-th derivatives of $f(t)$ satisfy

(1)

$$
G\left(f^{\prime}\right)=\frac{1}{u} G(f)-u^{\alpha} f(0)
$$

(2)

$$
G\left(f^{\prime \prime}\right)=\frac{1}{u^{2}} G(f)-\frac{1}{u} f(0) u^{\alpha}-f^{\prime}(0) u^{\alpha}
$$

(3)

$$
\begin{aligned}
G\left(f^{(n)}\right)= & \frac{1}{u^{n}} G(f)-\frac{1}{u^{n-1}} f(0) u^{\alpha}-\frac{1}{u^{n-2}} f^{\prime}(0) u^{\alpha} \\
& -\cdots-f^{(n-1)}(0) u^{\alpha}
\end{aligned}
$$

(4) Let $f(t)$ be piecewise continuous for $t \geq 0$ and integrable. Then

$$
G\left[\int_{0}^{t} f(\tau) d \tau\right]=u F(u)
$$

holds for $F(u)=G[f(t)]$.

Proof. By the integration by parts,

$$
\begin{aligned}
G\left(f^{\prime}\right) & =u^{\alpha} \int_{0}^{\infty} e^{-t / u} f^{\prime}(t) d t \\
& =u^{\alpha}\left[e^{-t / u} f(t)\right]_{0}^{\infty}+u^{\alpha-1} \int_{0}^{\infty} e^{-t / u} f(t) d t \\
& =\frac{1}{u} G(f)-u^{\alpha} f(0)
\end{aligned}
$$

and, similarly,

$$
\begin{aligned}
G\left(f^{\prime \prime}\right) & =\frac{1}{u} G\left(f^{\prime}\right)-f^{\prime}(0) u^{\alpha} \\
& =\frac{1}{u}\left[\frac{1}{u} G(f)-u^{\alpha} f(0)\right]-f^{\prime}(0) u^{\alpha} \\
& =\frac{1}{u^{2}} G(f)-\frac{1}{u} f(0) u^{\alpha}-f^{\prime}(0) u^{\alpha}
\end{aligned}
$$

follows.

Continuing this process by substitution as the above and using induction, (3) follows. Let us minutely establish the validity of the statement of (3) by the mathematical induction. For $n=1$, it clearly follows. Next, we suppose that

$$
\begin{aligned}
G\left(f^{(n)}\right)= & \frac{1}{u^{n}} G(f)-\frac{1}{u^{n-1}} f(0) u^{\alpha}-\frac{1}{u^{n-2}} f^{\prime}(0) u^{\alpha} \\
& -\cdots-f^{(n-1)}(0) u^{\alpha}
\end{aligned}
$$

and we show that $G\left(f^{(n+1)}\right)$ can be expressed by

$$
\begin{aligned}
G\left(f^{(n+1)}\right)= & \frac{1}{u^{n+1}} G(f)-\frac{1}{u^{n}} f(0) u^{\alpha} \\
& -\frac{1}{u^{n-1}} f^{\prime}(0) u^{\alpha}-\cdots-f^{(n)}(0) u^{\alpha} .
\end{aligned}
$$

In statement (1), the proof of statement (3) follows by applying (1) to $f^{(n)}$.

Finally, (4) follows as below.

Show that $G\left[\int_{0}^{t} f(\tau) d \tau\right]=u F(u)$ holds for $t>0$. Using Theorem 3 and putting $g(t)=\int_{0}^{t} f(\tau) d \tau$,

$$
\begin{aligned}
G(f(t)) & =G\left(g^{\prime}(t)\right)=\frac{1}{u} G(g)-u^{\alpha} g(0) \\
& =\frac{1}{u} G(g) .
\end{aligned}
$$

Moreover, $F(u)$ clearly satisfies a growth restriction. 
Let us check an example in [1] by the G-transform.

Example 4. Solve $y^{\prime \prime}-y=t, y(0)=1$, and $y^{\prime}(0)=1$.

Solution. Taking G-transform on both sides, we have

$$
\frac{1}{u^{2}} G(f)-\frac{1}{u} y(0) u^{\alpha}-y^{\prime}(0) u^{\alpha}-G(f)=u^{\alpha+2} .
$$

Organizing this equality, we have

$$
\left(\frac{1}{u^{2}}-1\right) G(f)=\left(u^{\alpha+2}+u^{\alpha}+u^{\alpha-1}\right) \text {. }
$$

Simplification gives

$$
\begin{aligned}
G(f) & =\left(u^{\alpha+2}+u^{\alpha}+u^{\alpha-1}\right) \cdot \frac{u^{2}}{1-u^{2}} \\
& =\frac{u^{\alpha+4}}{1-u^{2}}+\frac{u^{\alpha+2}}{1-u^{2}}+\frac{u^{\alpha+1}}{1-u^{2}} .
\end{aligned}
$$

Since

$$
\frac{u^{\alpha+4}}{1-u^{2}}=-u^{\alpha+2}+\frac{u^{\alpha+2}}{1-u^{2}},
$$

from Table 1, we have the solution

$$
y(t)=-t+2 \sin h t+\cos h t
$$

for $h$ is hyperbolic functions. From the substitution, above $y(t)$ is exactly a solution of the given equation. Of course, by a simple calculation, the above answer is equal to the solution of [1] which is $e^{t} \sin h t-t$.

2.3. Convolution and Integral Equations for G-Transform. It is a well-known fact that $\mathfrak{E}(f g) \neq \mathfrak{E}(f) \mathfrak{E}(g)$ and $\mathfrak{E}(f) \mathfrak{E}(g)=$ $\mathfrak{E}(f * g)$ for $f * g$ is the convolution of $f$ and $g$. This means that convolution has to do with the multiplication of transforms in Laplace transform. Here, we investigate the change at $G$-transform. If two functions $f$ and $g$ are integrable, the following theorem is held.

Lemma 5 (Lebesgue's dominated convergence theorem (LDCT) [14]). Let $(X, M, \mu)$ be a measure space and suppose that $\left\{f_{n}\right\}$ is a sequence of extended real-valued measurable functions defined on $X$ such that

(a) $\lim _{n \rightarrow \infty} f_{n}(x)=f(x)$ exists $\mu$-a.e.

(b) there is an integrable function $g$ so that, for each $n$, $\left|f_{n}\right| \leq g \mu$-a.e. Then $f$ is integrable and

$$
\lim _{n \rightarrow \infty} \int_{X} f_{n} d \mu=\int_{X} f d \mu
$$

We note that the above lemma gives validity to the following equality:

$$
\int \sum_{n=1}^{\infty} g_{n} d \mu=\sum_{n=1}^{\infty} \int g_{n} d \mu
$$

for $\left(g_{n}\right)$ is a nondecreasing sequence.
Theorem 6.

$$
u^{\alpha} G(f * g)=G(f) G(g)
$$

Proof. Let us put

$$
G(f)=u^{\alpha} \int_{0}^{\infty} e^{-\tau / u} f(\tau) d \tau
$$

and put

$$
G(g)=u^{\alpha} \int_{0}^{\infty} e^{-v / u} g(v) d v
$$

Then

$$
\begin{aligned}
G(f) G(g)= & u^{\alpha} \int_{0}^{\infty} e^{-\tau / u} f(\tau) \\
& \cdot u^{\alpha} \int_{0}^{\infty} e^{-v / u} g(v) d v d \tau .
\end{aligned}
$$

Let us put $t=v+\tau$, where $\tau$ is at first constant. Then $v=t-\tau$ and so we get

$$
\begin{aligned}
G(g) & =u^{\alpha} \int_{\tau}^{\infty} e^{-(t-\tau) / u} g(t-\tau) d t \\
& =e^{\tau / u} u^{\alpha} \int_{\tau}^{\infty} e^{-t / u} g(t-\tau) d t .
\end{aligned}
$$

Since the function $f$ is integrable, we can change the order of integration by using Lebesgue's dominated convergence theorem. Hence

$$
\begin{aligned}
G(f) G(g)= & u^{\alpha} \\
& \cdot u^{\alpha} \int_{0}^{\infty} \int_{\tau}^{\infty} f(\tau) e^{-t / u} g(t-\tau) d t d \tau,
\end{aligned}
$$

and when $t$ varies $\tau$ to $\infty, \tau$ varies 0 to $t$. Hence

$$
\begin{aligned}
G(f) G(g)= & u^{\alpha} \\
& \cdot u^{\alpha} \int_{0}^{\infty} e^{-t / u} \int_{0}^{t} f(\tau) g(t-\tau) d \tau d t \\
& =u^{\alpha} \cdot u^{\alpha} \int_{0}^{\infty} e^{-t / u}(f * g)(t) d t \\
& =u^{\alpha} G(f * g) .
\end{aligned}
$$

It is a well-known fact that convolution helps us to solve integral equations of certain type, mainly Volterra integral equation. Hence, we would like to check the theorem by means of some examples using $G$-transform.

Example 7. Solve the Volterra integral equation of the second kind

$$
y(t)-\int_{0}^{t} y(\tau) \sin (t-\tau) d \tau=t .
$$


Solution. This is rewritten as a convolution:

$$
y(t)-y * \sin t=t
$$

Taking G-transform on both sides and applying Theorem 6, we have

$$
\begin{aligned}
& Y(u)-u^{-\alpha} Y(u) \frac{u^{\alpha+2}}{1+u^{2}}=Y(u)\left(1-\frac{u^{2}}{1+u^{2}}\right) \\
& =u^{\alpha+2}
\end{aligned}
$$

for $Y=G(y)$. The solution is

$$
Y(u)=\left(1+u^{2}\right) u^{\alpha+2}
$$

and gives the answer

$$
y(t)=t+\frac{1}{6} t^{3}
$$

by Table 1 .

Example 8. Solve the Volterra integral equation of the second kind

$$
y(t)-\int_{0}^{t}(1+\tau) y(t-\tau) d \tau=1-\sinh t
$$

Solution. In a way similar to Example 7, the given equation is same as $y-(1+t) * y=1-\sinh t$. Taking $G$-transform, we have

$$
Y(u)-u^{-\alpha}\left(u^{\alpha+1}+u^{\alpha+2}\right) Y(u)=u^{\alpha+1}-\frac{u^{\alpha+2}}{1-u^{2}}
$$

hence,

$$
Y(u)\left[1-u-u^{2}\right]=\frac{u^{\alpha+1}\left(1-u-u^{2}\right)}{1-u^{2}} .
$$

Simplification gives

$$
Y(u)=\frac{u^{\alpha+1}}{1-u^{2}}
$$

and so we obtain the answer

$$
y(t)=\cosh t
$$

by Table 1 .

Example 9. Find the solution of

$$
y(t)+\int_{0}^{t}(t-\tau) y(\tau) d \tau=1
$$

Solution. Since this equation is $y+y * t=1$, taking $G^{-}$ transform on both sides, we have

$$
Y+u^{-\alpha}\left(Y \cdot u^{\alpha+2}\right)=u^{\alpha+1}
$$

for $Y=G(y)$. Thus

$$
Y=\frac{u^{\alpha+1}}{1+u^{2}}
$$

and so we obtain the solution $y=\cos t$.

Let us check this by the direct calculation. Expanding the given equation, we have

$$
y(t)+t \cdot \int_{0}^{t} y(\tau) d \tau-\int_{0}^{t} \tau y(\tau) d \tau=1 .
$$

Differentiating both sides twice with respect to $t$, we have $y^{\prime \prime}(t)+y(t)=0$. Thus, from $y(0)=1$ and $y^{\prime}(0)=0$ obtained by calculating course, we have the solution $y=\cos t$.

Similarly, we can easily obtain the solution of integral equations by using $\alpha=-2$. For example, let us consider

$$
y(t)-\int_{0}^{t} y(\tau) d \tau=1
$$

By the $G$-transform, we have

$$
Y-u Y=\frac{1}{u}
$$

and so, we have the solution $y=e^{t}$ for $Y=G(y)$. Of course, this result is the same as the result

$$
y-y * 1=1
$$

by using convolution, and the result of the direct calculation

$$
y^{\prime}(t)-y(t)=0
$$

is so as well. Similarly, since

$$
G\left(t e^{a t}\right)=\frac{u^{\alpha+2}}{(1-a u)^{2}}
$$

the solution of

$$
y(t)+2 e^{t} \int_{0}^{t} e^{-\tau} y(\tau) d \tau=t e^{t}
$$

is $y=\sinh t$. Here, we note that the $G$-transform of $y+2(y *$ $\left.e^{t}\right)=t e^{t}$ is

$$
Y+2 u^{-\alpha} Y \cdot \frac{u^{\alpha+1}}{u(1-u)}=\frac{u^{\alpha+2}}{(1-u)^{2}}
$$

for $Y=G(y)$.

\section{Differentiation and Integration of Transforms: ODEs with Variable Coefficients}

Theorem 10. Let us put $Y=F(u)$. Then

(1) $F^{\prime}(u)=Y\left(\alpha / u+t / u^{2}\right)$,

(2) $F^{\prime \prime}(u)=Y\left(\left(\alpha^{2}-\alpha\right) / u^{2}+2 t(\alpha-1) / u^{3}+t^{2} / u^{4}\right)$, 
(3) $\int_{u}^{\infty} F(s) d s=\int_{0}^{\infty} f(t) \int_{u}^{\infty} s^{\alpha} e^{-t / s} d s d t$,

(4) $G(t y)=-\alpha u Y-(d / d u) G(y), G\left(t y^{\prime}\right)=-\alpha u[(1 / u) Y-$ $\left.y(0) u^{\alpha}\right]-(d / d u) G\left(y^{\prime}\right)$,

(5) $G\left(t y^{\prime \prime}\right)=-\alpha u\left[\left(1 / u^{2}\right) Y-(1 / u) y(0) u^{\alpha}-y^{\prime}(0) u^{\alpha}\right]-$ $(d / d u) G\left(y^{\prime \prime}\right)$.

Proof. (1) Since

$$
\begin{aligned}
F(u)= & G(f)=u^{\alpha} \int_{0}^{\infty} e^{-t / u} f(t) d t \\
F^{\prime}(u)= & \alpha u^{\alpha-1} \int_{0}^{\infty} e^{-t / u} f(t) d t \\
& +u^{\alpha} \frac{t}{u^{2}} \int_{0}^{\infty} e^{-t / u} f(t) d t=Y\left(\frac{\alpha}{u}+\frac{t}{u^{2}}\right)
\end{aligned}
$$

for $Y=F(u)$.

(2) Since

$$
F^{\prime \prime}(u)=\frac{d}{d u} F^{\prime}(u)=\frac{d}{d u}\left[Y\left(\frac{\alpha}{u}+\frac{t}{u^{2}}\right)\right],
$$

we have

$$
\begin{aligned}
F^{\prime \prime}(u) & =Y^{\prime}\left(\frac{\alpha}{u}+\frac{t}{u^{2}}\right)+Y\left(\frac{\alpha}{u}+\frac{t}{u^{2}}\right)^{\prime} \\
& =Y\left(\frac{\alpha}{u}+\frac{t}{u^{2}}\right)^{2}-Y\left(\frac{\alpha}{u^{2}}+\frac{2 t}{u^{3}}\right) .
\end{aligned}
$$

Organizing this equality, we have

$$
F^{\prime \prime}(u)=Y\left(\frac{\alpha^{2}-\alpha}{u^{2}}+\frac{2 t(\alpha-1)}{u^{3}}+\frac{t^{2}}{u^{4}}\right) .
$$

(3)

$$
\begin{aligned}
\int_{u}^{\infty} F(s) d s & =\int_{u}^{\infty}\left[s^{\alpha} \int_{0}^{\infty} e^{-t / s} f(t) d t\right] d s \\
& =\int_{u}^{\infty} \int_{0}^{\infty} s^{\alpha} e^{-t / s} f(t) d t d s \\
& =\int_{0}^{\infty} f(t) \int_{u}^{\infty} s^{\alpha} e^{-t / s} d s d t
\end{aligned}
$$

thanks to Lebesgue's dominated convergence theorem.

(4) In the definition of

$$
F(u)=G(y)=u^{\alpha} \int_{0}^{\infty} e^{-t / u} y(t) d t,
$$

let us put $1 / u=s$. Then

$$
\begin{aligned}
F(u)= & G(y)=\frac{1}{s^{\alpha}} \int_{0}^{\infty} e^{-s t} y(t) d t \\
F^{\prime}(u)= & -\alpha s^{-\alpha-1} \int_{0}^{\infty} e^{-s t} y(t) d t \\
& -\frac{1}{s^{\alpha}} \int_{0}^{\infty} e^{-s t} t y(t) d t=\frac{-\alpha}{s} G(y)-G(t y) .
\end{aligned}
$$

Since $1 / s$ is the same as $u$, we have

$$
G(t y)=-\alpha u Y-F^{\prime}(u)=-\alpha u Y-\frac{d}{d u} G(y)
$$

for $Y=G(y)$.

In the above theorem, we note that $G\left(t y^{(n)}\right)$ can be represented by

$$
G\left(t y^{(n)}\right)=-\alpha u G\left(y^{(n)}\right)-\frac{d}{d u} G\left(y^{(n)}\right) .
$$

(5) From (4), the statement is held for an arbitrary integer $n$.

\section{G-Transforms of Heaviside \\ Function and Dirac's Delta Function: Shifted Data Problems}

\subsection{Heaviside Function}

$$
\begin{aligned}
G[h(t-a)] & =u^{\alpha} \int_{0}^{\infty} e^{-t / u} h(t-a) d t \\
& =u^{\alpha} \int_{a}^{\infty} e^{-t / u} d t=-u^{\alpha+1}\left[e^{-t / u}\right]_{a}^{\infty} \\
& =u^{\alpha+1} e^{-a / u},
\end{aligned}
$$

where $h$ is Heaviside function.

4.2. Dirac's Delta Function. We consider the function $f_{k}(t-$ $a)=1 / k$ if $a \leq t \leq a+k$ and 0 otherwise. Taking $G$-transform, we have

$$
\begin{aligned}
G\left[f_{k}(t-a)\right] & =u^{\alpha} \int_{0}^{\infty} e^{-t / u} f_{k}(t-a) d t \\
& =-\frac{u^{\alpha+1}}{k}\left[e^{-t / u}\right]_{a}^{a+k} \\
& =-\frac{u^{\alpha+1}}{k}\left(e^{-(a+k) / u}-e^{-a / u}\right) \\
& =-\frac{u^{\alpha+1}}{k} e^{-a / u} \cdot\left(e^{-k / u}-1\right) .
\end{aligned}
$$

If we denote the limit of $f_{k}$ as $\delta(t-a)$, then

$$
\delta(t-a)=\lim _{k \rightarrow 0} f_{k}(t-a)=u^{\alpha} e^{-a / u}
$$

4.3. Shifted Data Problems. For given differential equations $y^{\prime \prime}+a y^{\prime}+b y=r(t), y\left(t_{0}\right)=c_{0}$, and $y^{\prime}\left(t_{0}\right)=c_{1}$, where $t_{0} \neq 0$ and $a$ and $b$ are constant, we can set $t=t_{1}+t_{0}$. Then $t=t_{0}$ gives $t_{1}=0$ and so we have

$$
\begin{aligned}
y_{1}^{\prime \prime}+a y_{1}^{\prime}+b y_{1} & =r\left(t_{1}+t_{0}\right), \\
y_{1}(0) & =c_{0}, \\
y_{1}^{\prime}(0) & =c_{1}
\end{aligned}
$$

for input $r(t)$. Taking the transform, we can obtain the output $y(t)$. 


\section{The Solution of Semi-Infinite String by $G$-Transform}

Let us check the solution of semi-infinite string by $G$ transform in terms of a typical example as given in [1].

Example 11 (semi-infinite string). Find the displacement $w(x, t)$ of an elastic string subject to the following conditions:

(a) The string is initially at rest on $x$-axis from $x=0$ to $\infty$.

(b) For $t>0$, the left end of the string is moved in a given fashion, namely, according to a single sine wave $w(0, t)=f(t)=\sin t$, if $0 \leq t \leq 2 \pi$ and zero otherwise.

(c) Furthermore, $\lim w(x, t)=0$ as $x \rightarrow \infty$ for $t \geq 0$.

Of course there is no infinite string, but our model describes a long string or rope (of negligible weight) with its right end fixed far out on $x$-axis [1].

Solution. It is a well-known fact that the equation of semiinfinite string can be expressed by

$$
\frac{\partial^{2} w}{\partial t^{2}}=c^{2} \frac{\partial^{2} w}{\partial x^{2}}
$$

subject to $w(0, t)=f(t), \lim w(x, t)=0$ as $x \rightarrow \infty, w(x, 0)=$ 0 , and $w_{t}(x, 0)=0$. Taking $G$-transform with respect to $t$ and by Theorem 3 , we have

$$
\begin{aligned}
G\left[\frac{\partial^{2} w}{\partial t^{2}}\right] & =\frac{1}{u^{2}} W-\frac{1}{u} w(x, 0) u^{\alpha}-w_{t}(x, 0) u^{\alpha} \\
& =\frac{1}{u^{2}} W
\end{aligned}
$$

for $W(x, u)=G[w(x, t)]$. On the other hand,

$$
\begin{aligned}
G\left[\frac{\partial^{2} w}{\partial x^{2}}\right] & =u^{\alpha} \int_{0}^{\infty} e^{-t / u} \frac{\partial^{2} w}{\partial x^{2}} d t \\
& =\frac{\partial^{2}}{\partial x^{2}} u^{\alpha} \int_{0}^{\infty} e^{-t / u} w(x, t) d t \\
& =\frac{\partial^{2}}{\partial x^{2}} G[w(x, t)]=\frac{\partial^{2} W}{\partial x^{2}}
\end{aligned}
$$

Thus, the equation $(*)$ becomes

$$
c^{2} \frac{\partial^{2} W}{\partial x^{2}}-\frac{1}{u^{2}} W=0 .
$$

Since this equation may be regarded as an ODE for $W(x, u)$ considered as a function of $x$, its general solution can be represented by

$$
W(x, u)=A(u) e^{x / c u}+B(u) e^{-x / c u} .
$$

From the initial conditions, we have $W(0, u)=G[w(0, t)]=$ $G[f(t)]=F(u)$. In $[15,16]$, we have dealt with the validity on exchangeability of integral and limit in the solving process of PDEs by using dominated convergence theorem. Hence, we have

$$
\begin{aligned}
\lim _{x \rightarrow \infty} W(x, u) & =\lim _{x \rightarrow \infty} u^{\alpha} \int_{0}^{\infty} e^{-t / u} w(x, t) d t \\
& =u^{\alpha} \int_{0}^{\infty} e^{-t / u} \lim _{x \rightarrow \infty} w(x, t) d t=0,
\end{aligned}
$$

implying that $A(u)=0$ and so $W(0, u)=B(u)=F(u)$. Thus,

$$
W(x, u)=F(u) e^{-x / c u} .
$$

By the $t$-shifting theorem (Theorem 2), we obtain the inverse transform

$$
w(x, t)=f\left(t-\frac{x}{c}\right) h\left(t-\frac{x}{c}\right)=\sin \left(t-\frac{x}{c}\right)
$$

for $x / c<t<x / c+2 \pi$ and zero otherwise, where $h$ is Heaviside function.

\section{Conclusion}

This paper has constructed the generalized form of Laplacetyped integral transforms and has established the properties of the generalized Laplace-typed integral transform, $G$ transform. The transform is comprehensive form, and it has been well adapted in a number of situations of engineering problems by choosing adequate values $\alpha$ in kernel, and we newly presented the value $\alpha=-2$ which is suitable for transforms of integrals. And the future work is to find the other values of $\alpha$ which are suitable for each situation. The strong point of this article is in the high applicability to engineering problems.

\section{Conflicts of Interest}

The author declares that there are no conflicts of interest regarding the publication of this paper.

\section{Acknowledgments}

This research was supported by Kyungdong University Research Grant.

\section{References}

[1] E. Kreyszig, Advanced Engineering Mathematics, Wiley, Singapore, 2013.

[2] G. K. Watugala, "Sumudu transform-a new integral transform to solve differential equations and control engineering problems," International Journal of Mathematical Education in Science and Technology, vol. 24, no. 1, pp. 35-43, 1993.

[3] T. M. Elzaki, S. M. Elzaki, and E. M. A. Hilal, "Elzaki and Sumudu transforms for solving some differential equations," Global Journal of Pure \& Applied Mathematics, vol. 8, no. 2, pp. 167-173, 2012.

[4] Hj. Kim, "The time shifting theorem and the convolution for Elzaki transform," Global Journal of Pure and Applied Mathematics, vol. 87, pp. 261-271, 2013. 
[5] F. B. Belgacem and S. Sivasundaram, "New developments in computational techniques and transform theory applications to nonlinear fractional and stochastic differential equations and systems," Nonlinear Studies. The International Journal, vol. 22, no. 4, pp. 561-563, 2015.

[6] F. B. M. Belgacem and A. Karaballi, "Sumudu transform fundamental properties investigations and applications," Journal of Applied Mathematics and Stochastic Analysis, vol. 2006, Article ID 91083, 23 pages, 2006.

[7] T. M. Elzaki and J. Biazar, "Homotopy perturbation method and Elzaki transform for solving system of nonlinear partial differential equations," World Applied Sciences Journal, vol. 24, no. 7, pp. 944-948, 2013.

[8] T. M. Elzaki and H. Kim, "The solution of radial diffusivity and shock wave equations by Elzaki variational iteration method," International Journal of Mathematical Analysis, vol. 9, no. 21-24, pp. 1065-1071, 2015.

[9] H. A. Agwa, F. M. Ali, and A. Kilicman, "A new integral transform on time scales and its applications," Advances in Difference Equations, vol. 60, pp. 1-14, 2012.

[10] H. Eltayeb and A. Kilicman, "On some applications of a new integral transform," International Journal of Mathematical Analysis, vol. 4, no. 1-4, pp. 123-132, 2010.

[11] H. Eltayeb, A. Kilicman, and M. B. Jleli, "Fractional Integral Transform and Application," Abstract and Applied Analysis, vol. 2015, Article ID 150863, 1 page, 2015.

[12] H. Kim, "The form of solution of ODEs with variable coefficients by means of the integral and Laplace transform," Global Journal of Pure and Applied Mathematics, vol. 12, pp. 2901-2904, 2016.

[13] H. Kim, "The shifted data problems by using transform of derivatives," Applied Mathematical Sciences, vol. 8, no. 149-152, pp. 7529-7534, 2014.

[14] D. L. Cohn, Measure Theory, Birkhäauser, Boston, Mass, USA, 1980.

[15] H. C. Chae and H. Kim, "The validity checking on the exchange of integral and limit in the solving process of PDEs," International Journal of Mathematical Analysis, vol. 8, no. 22, pp. 10891092, 2014.

[16] J. Jang and H. Kim, "An application of monotone convergence theorem in pdes and fourier analysis," Far East Journal of Mathematical Sciences, vol. 98, no. 5, pp. 665-669, 2015. 


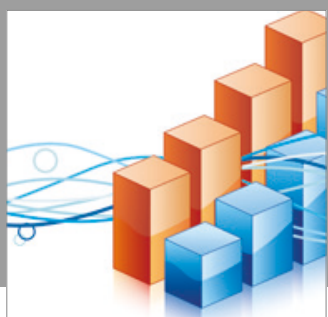

Advances in

Operations Research

vatersals

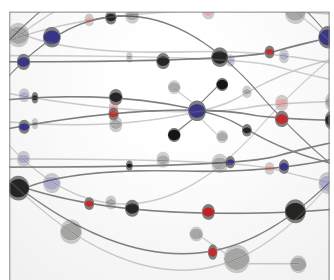

\section{The Scientific} World Journal
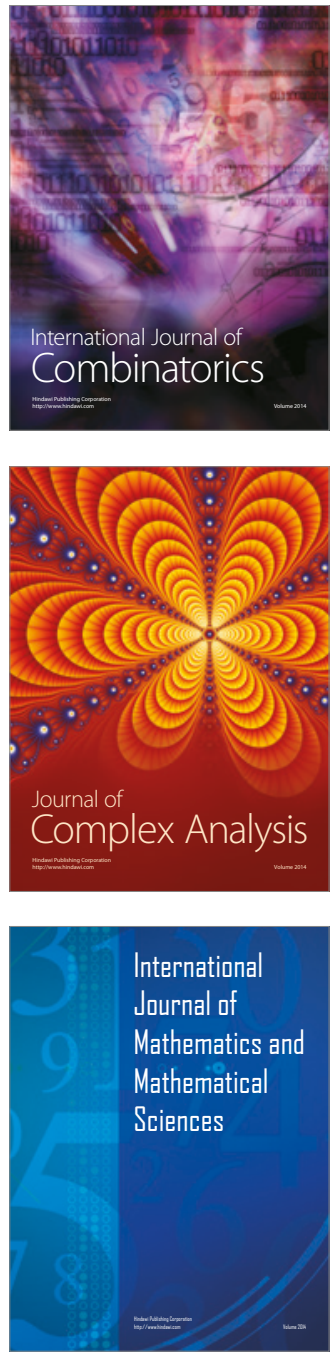
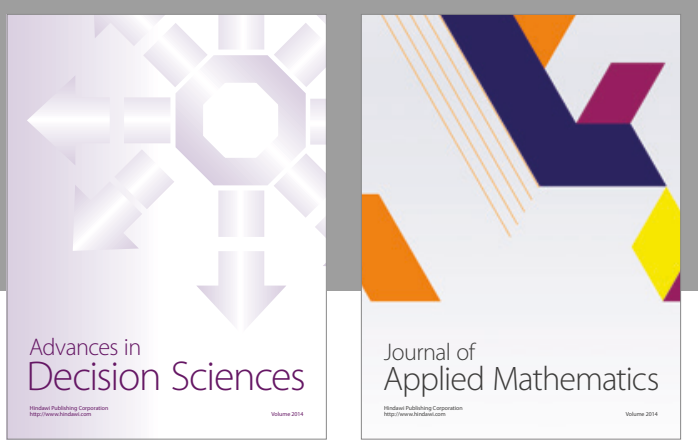

Algebra

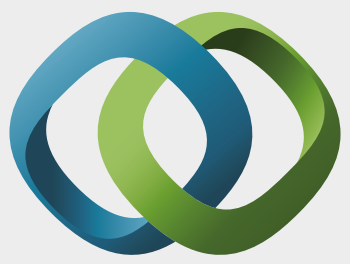

\section{Hindawi}

Submit your manuscripts at

https://www.hindawi.com
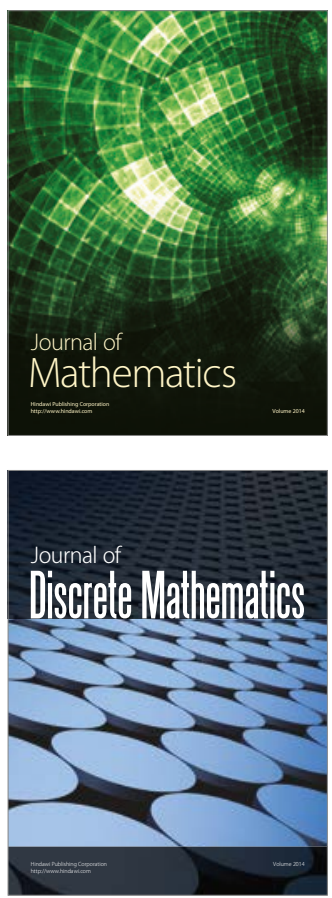

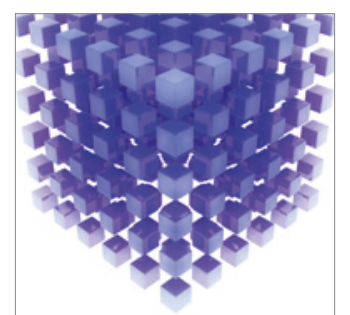

Mathematical Problems in Engineering
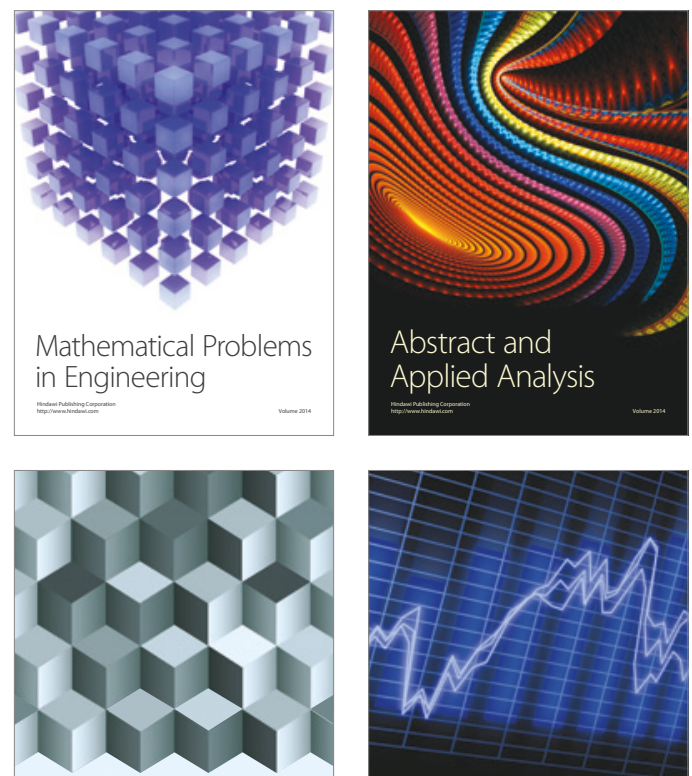

Journal of

Function Spaces

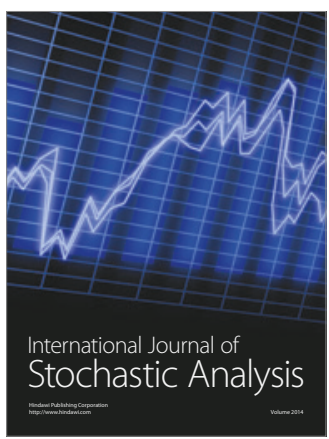

Probability and Statistics
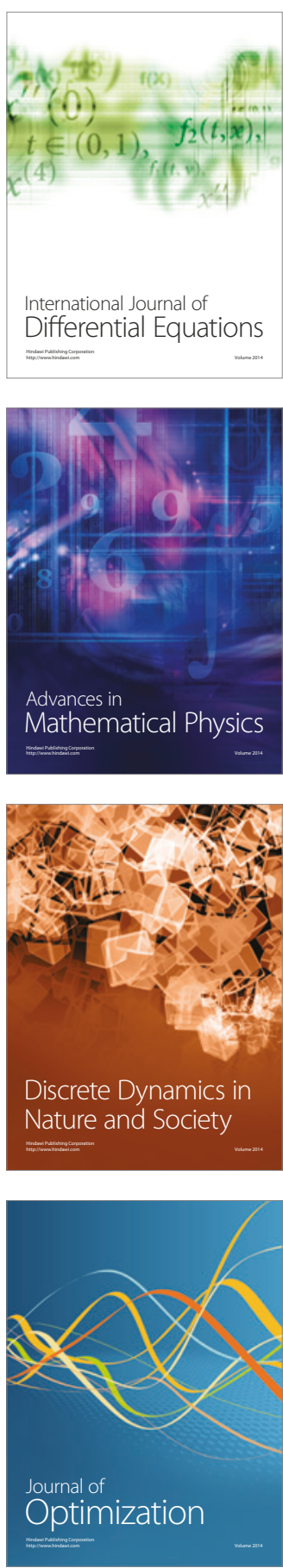